\title{
Multiple Rising Doses of Oral BI 425809, a GlyT1 Inhibitor, in Young and Elderly Healthy Volunteers: A Randomised, Double-Blind, Phase I Study Investigating Safety and Pharmacokinetics
}

\author{
Viktoria Moschetti $^{1}$ - Christina Schlecker ${ }^{1}$ Sven Wind ${ }^{2} \cdot$ Sophia Goetz $^{2} \cdot$ Holger Schmitt $^{2}$. \\ Armin Schultz $^{3} \cdot$ Karl-Heinz Liesenfeld ${ }^{2} \cdot$ Glen Wunderlich ${ }^{4} \cdot$ Michael Desch $^{2}$
}

Published online: 30 May 2018

(C) The Author(s) 2018

\begin{abstract}
Background and Objective Schizophrenia and Alzheimer's disease are characterised by abnormalities in glutamatergic pathways related to $N$-methyl-D-aspartate receptor hypofunction. Glycine is an $\mathrm{N}$-methyl-D-aspartate receptor coagonist; inhibition of glycine transporter 1 may improve $\mathrm{N}$-methyl-D-aspartate receptor function. This phase I, randomised, two-part study evaluated the safety, tolerability and pharmacokinetic profile of BI 425809, a novel glycine transporter 1 inhibitor, in healthy male and female volunteers.

Methods Part 1 evaluated BI 425809 10, 25, 50 or $75 \mathrm{mg}$ once daily or $75 \mathrm{mg}$ twice daily in young subjects, and $25 \mathrm{mg}$ or $50 \mathrm{mg}$ once daily in elderly subjects. Each dose group comprised 12 subjects who received BI 425809 $(n=9)$ or placebo $(n=3)$ for 14 days (day 1: single dose; days 4-14: multiple dosing). Part 2 compared
\end{abstract}

Viktoria Moschetti and Christina Schlecker contributed equally to this work.

Electronic supplementary material The online version of this article (https://doi.org/10.1007/s40261-018-0660-2) contains supplementary material, which is available to authorized users.

Michael Desch

michael.desch@boehringer-ingelheim.com

1 Boehringer Ingelheim International $\mathrm{GmbH}$, Ingelheim am Rhein, Germany

2 Boehringer Ingelheim International $\mathrm{GmbH}$, Birkendorfer Str. 65, 88397 Biberach an der Riss, Germany

3 CRS Clinical Research Services Mannheim GmbH, Mannheim, Germany

4 Boehringer Ingelheim (Canada) Ltd., Burlington, ON, Canada pharmacokinetic profiles in 12 subjects who received a single dose of BI $42580925 \mathrm{mg}$ in the morning and evening.

Results Pharmacokinetic profiles were similarly shaped for all dose groups. Median time to maximum plasma concentration was 3.0-4.5 h with steady state being reached between days 6 and 10. Pharmacokinetic parameters demonstrated dose linearity at the predicted therapeutic exposure range of BI $425809 \leq 25 \mathrm{mg}$ once daily, but increased less than dose proportionally for $\geq 50 \mathrm{mg}$ once daily. All reported adverse events were of mild-to-moderate intensity, 51/84 (61\%; part 1) subjects had one or more treatment-related adverse event, no serious adverse events occurred and no dose dependency was observed.

Conclusions Pharmacokinetic properties support both morning and evening dosing. BI 425809 was generally well tolerated at all tested doses.

Clinicaltrials.gov identifier NCT02337283.

\section{Key Points}

BI 425809 was well tolerated within the tested dose range for all subjects (healthy young and elderly), with reported adverse events being of mild-tomoderate intensity.

Within the predicted therapeutic exposure range of BI $425809 \leq 25 \mathrm{mg}$ once daily, pharmacokinetic results demonstrated dose linearity and supported both morning and evening dosing regimens. 


\section{Introduction}

Cognitive impairment is a significant feature of neurodegenerative conditions, such as Alzheimer's disease (AD), and psychiatric conditions, including schizophrenia [1-4]. These conditions are underpinned by abnormalities in glutamatergic pathways related to $N$-methyl-D-aspartate (NMDA) receptor hypofunction in cortical and hippocampal brain areas. Such abnormalities have been associated with the cognitive impairment experienced by these patients [5, 6]. Glycine is an NMDA receptor coagonist; inhibition of glycine transporter 1 (GlyT1), which regulates synaptic glycine levels, may therefore improve NMDA receptor function by raising glycine levels in the synaptic cleft [7]. Animal data indicate that improved NMDA receptor signalling leads to an increase in longterm potentiation and synaptic plasticity [8], which improves cognitive function and memory [9].

BI 425809, a potent and selective GlyT1 inhibitor, is a new chemical entity hypothesised to improve cognitive function and memory in patients with schizophrenia and AD. A phase I, first-in-human, single rising dose study found single doses of BI 425809 up to $100 \mathrm{mg}$, administered as an oral solution, to be generally well tolerated in young healthy male volunteers [10].

A pharmacokinetic analysis of BI 425809 supported a once-daily (QD) dosing regimen for future multiple-dose studies [10]. Plasma concentration-time profiles were similarly shaped at all dose levels $(0.5-150 \mathrm{mg})$ and were characterised by rapid absorption (median time to maximum plasma concentration of $\sim 45 \mathrm{~min}$ ) followed by a biphasic distribution phase. Dose proportionality was observed in all exposure parameters [area under the concentration-time curve over the time interval from 0 to the last quantifiable data point $\left(\mathrm{AUC}_{0-t z}\right)$, area under the concentration-time curve over the time interval from 0 extrapolated to infinity $\left(\mathrm{AUC}_{0-\infty}\right)$, maximum plasma concentration $\left.\left(C_{\max }\right)\right]$ across the entire dose range. The geometric mean (gMean) terminal half-life was not dose dependent, ranging between 32.5 and $47.0 \mathrm{~h}$ [10]. The bioavailability of BI 425809 administered as a tablet was lower than the bioavailability of BI 425809 administered as an oral solution. Adjusted gMean ratios for $\mathrm{AUC}_{0-t z}$ and $C_{\max }$ for BI 425809 administered as a tablet fasted vs. oral solution fasted were $80.5 \%$ [90\% confidence interval (CI) 74.0-87.6) and 50.0\% (90\% CI 45.1-55.4), respectively. Furthermore, the single rising dose study identified a moderate increase in BI 425809 bioavailability following administration as a tablet under fed conditions (high-fat/ high-calorie breakfast of $\sim 1000 \mathrm{kcal}$ ) vs. strongly fasted conditions [adjusted gMean ratios: $\mathrm{AUC}_{0-t z}$ 125.9\% (90\% CI 115.7-137.0); $C_{\max } 142.1 \%$ [90\% CI 128.3-157.4)] [10].
The current study extends this investigation to include less extreme meal conditions (standard-fat/standard-calorie meal of $\sim 500 \mathrm{kcal}$ ) that are more representative of daily life. A recent drug-drug interaction study confirmed invitro data indicating that cytochrome P450 (CYP) $3 \mathrm{~A} 4$ is a major component of the BI 425809 metabolic pathway [11].

The primary objective of this study was to evaluate the safety and tolerability of multiple rising doses of BI 425809, compared with placebo, in healthy young and elderly subjects. Furthermore, BI 425809 pharmacokinetic parameters were determined to explore dose proportionality, attainment of steady state and the effect of a standardised light breakfast on BI 425809 steady-state exposure. Finally, this study additionally investigated differences in the pharmacokinetics, safety and tolerability of BI 425809 when administered in the morning vs. evening, to evaluate whether an evening dosing regimen, which may attenuate sedative or visual adverse events (AEs), would be beneficial for subjects in phase II trials.

\section{Subjects and Methods}

\subsection{Ethical Conduct}

All study procedures, protocols and documents met with institutional approval following review by the study centre's independent ethics committee. The study was conducted in accordance with the clinical study protocol, the International Conference for Harmonisation of Technical Requirements for Pharmaceuticals for Human Use Good Clinical Practice guidelines [12] and local legislation, in accordance with the principles of the Declaration of Helsinki [13]. All subjects provided signed and dated written informed consent prior to study procedures.

\subsection{Study Design}

This was a phase I study conducted at the Human Pharmacology Unit CRS Clinical Research Services in Mannheim, Germany (Clinicaltrials.gov identifier: NCT02337283), consisting of two parts. The first part was randomised, double blinded, and placebo controlled within parallel-dose groups, and aimed to assess the safety, tolerability and pharmacokinetics following multiple doses of BI 425809 10-75 mg, in healthy young and healthy elderly subjects compared with placebo. The second part of this study was randomised and open-label, with a two-treatment, two-sequence, two-period crossover, and aimed to assess the pharmacokinetics, safety and tolerability of single BI $42580925-\mathrm{mg}$ doses given in the morning or evening in healthy young subjects. An overview of the study design is given in Fig. 1. 


\subsubsection{Part 1: Multiple-Dose Component}

Subjects were allocated to seven sequential dose groups; five groups comprised healthy young subjects [BI 425809 QD at $10,25,50$ or $75 \mathrm{mg}$, or BI 425809 twice daily (BID) at $75 \mathrm{mg}$ ], and two comprised healthy elderly subjects (BI 425809 QD at 25 or $50 \mathrm{mg}$ ). Within each dose group, subjects were randomised to receive the active drug or placebo at a ratio of $3: 1$, such that nine subjects received the active drug and three received placebo. Each subject received a single dose of BI 425809 or placebo on day 1 , followed by QD (dose groups 1-6, 10-75 mg) or BID (dose group $8,75 \mathrm{mg}$ ) dosing for 10 days from day 4 onwards. On day 14, all dose groups received one single dose of BI 425809 or placebo. Dose groups were investigated consecutively in ascending order and a documented safety review was undertaken prior to each dose escalation. Healthy elderly subjects were dosed after a respective dose of BI 425809 had been well tolerated in healthy young subjects. Blood samples for pharmacokinetic assessments were taken following the first and last doses, and at regular intervals between days 1 and 14, after which daily samples were collected until day 23. Urine was collected for pharmacokinetic analysis during pre-specified periods following the first and last doses in the QD dose groups only. The effects of different types of food intake on BI 425809 pharmacokinetics were analysed in dose groups 2 and 5 (BI $42580925 \mathrm{mg}$ in young and elderly subjects, respectively) under light-fed (standardised light breakfast [ $\sim 500 \mathrm{kcal}]$ served $30 \mathrm{~min}$ before BI 425809 administration; day 12, dose group 2) [Table 1 of the Electronic Supplementary Material (ESM)], and light-fasted (no food

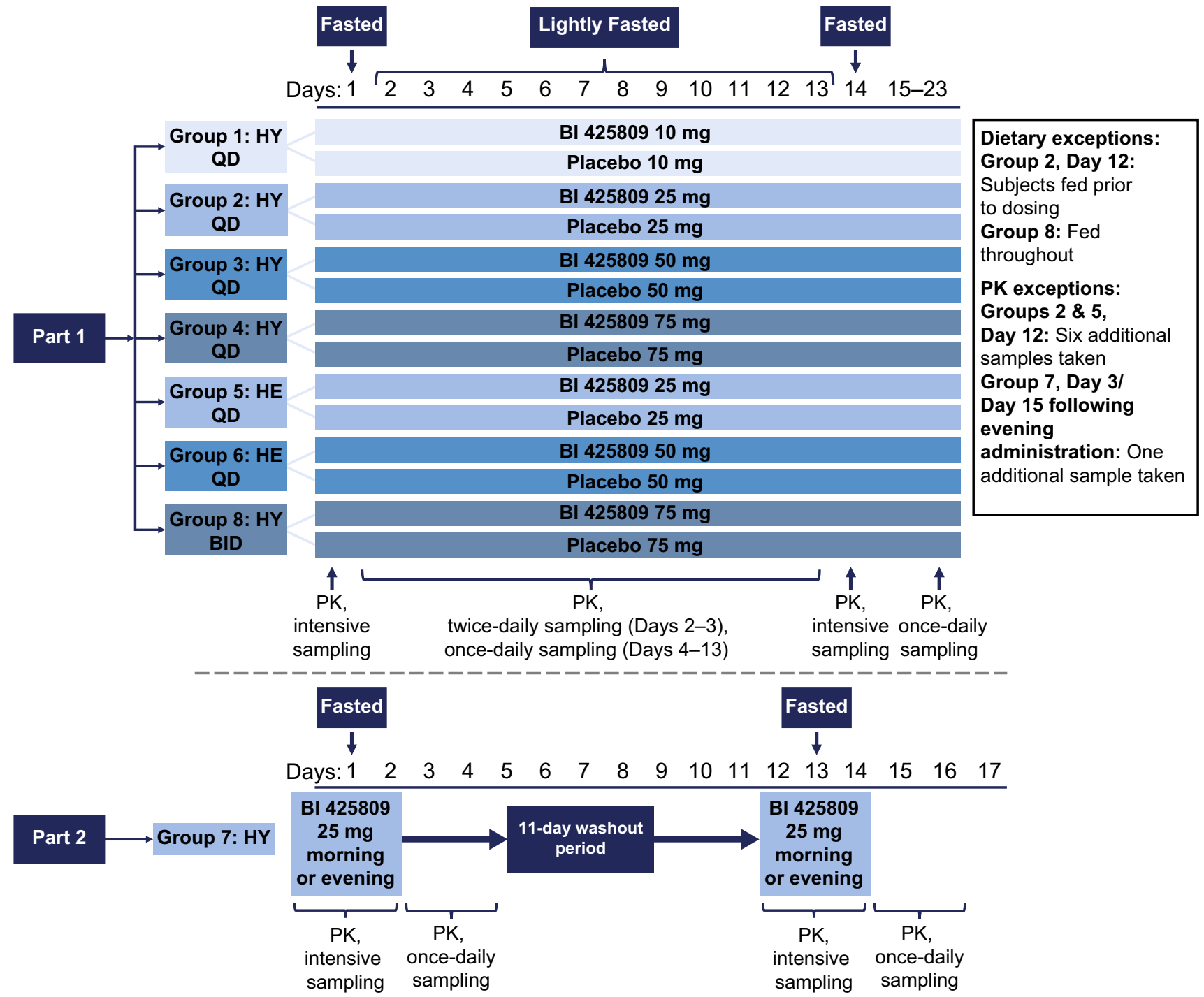

Fig. 1 Study design diagram. $B I D$ twice daily, $H E$ healthy elderly, $H Y$ healthy young, $P K$ pharmacokinetics, $Q D$ once daily 
for $\geq 10 \mathrm{~h}$ before, until $1 \mathrm{~h}$ after BI 425809 administration; day 12, dose group 5) conditions. In both dose groups, additional pharmacokinetic profiles were taken on day 12 and compared with the respective pharmacokinetic profiles on day 14. Fasted conditions (no food for $\geq 10 \mathrm{~h}$ before, until $3.5 \mathrm{~h}$ after BI 425809 administration) were employed on days 1 and 14, with light-fasted conditions used for the remaining treatment days in all groups except group 8 (Fig. 1). Twice-daily dosing and administration under light-fed conditions were applied to dose group 8 to increase the bioavailability of BI 425809 .

\subsubsection{Part 2: Morning vs. Evening Dosing}

Each subject (dose group 7) received two single doses of BI $42580925 \mathrm{mg}$ separated by a washout period of at least 11 days. The dose of BI 425809 was administered either in the morning or in the evening, per the randomisation scheme. All subjects were in a fasted state for the administration of BI 425809. Blood samples for pharmacokinetic assessment were taken prior to and up to $96 \mathrm{~h}$ after administration of the active drug (Fig. 1).

\subsection{Subjects}

\subsubsection{Inclusion Criteria}

A total of 96 subjects were entered into the study: 84 in part 1 , of whom 24 were elderly subjects, and 12 in part 2 . Eligible subjects were healthy male or female (of nonchildbearing potential) volunteers, 18-50 years of age for young subjects, or 65-80 years of age for elderly subjects, with a body mass index of $18.5-29.9 \mathrm{~kg} / \mathrm{m}^{2}$.

\subsubsection{Exclusion Criteria}

Subjects were excluded if they exhibited any evidence of a concomitant disease judged as clinically relevant upon physical examination, vital-sign assessment, electrocardiogram (ECG) or laboratory test (haematology, clinical chemistry, infectious serology and urinalysis). Other key exclusion criteria included: gastrointestinal, hepatic, renal, respiratory, cardiovascular, metabolic, immunological or hormonal disorders; diseases of the central nervous system, other neurological disorders, or psychiatric disorders; history of suicidal behaviour, macular degeneration or relevant orthostatic hypotension, fainting spells or blackouts; chronic or relevant acute infections; history of relevant allergy/hypersensitivity (including allergy to the study medication or its excipients); drug abuse, excessive alcohol intake ( $>20 \mathrm{~g} /$ day for female individuals and $>30 \mathrm{~g} /$ day for male individuals), use of tobacco (more than five cigarettes, one cigar or one pipe per day) or inability to refrain from smoking on study days.

\subsection{Treatments}

BI 425809 and placebo tablets were manufactured by Boehringer Ingelheim International $\mathrm{GmbH}$, Germany. Subjects received either placebo or BI 425809 as 5- or 25-mg tablets according to the dose strength required by the dosing group assigned. To maintain blinding, placebo tablets were matched in size, colour and shape to BI 425809 tablets (white to off-white; round; $5 \mathrm{mg}$, $\sim 6 \mathrm{~mm}$ in diameter; $25 \mathrm{mg}, \sim 10 \mathrm{~mm}$ in diameter). The tablet formulation was considered appropriate based on the planned dosing, similarity to the intended market formulation and the expectation that sufficient exposure would be obtained to meet the study aims.

\subsection{Study Endpoints}

\subsubsection{Safety Assessments}

The primary endpoint of part 1 and the secondary endpoint of part 2 was the number $(N[\%])$ of subjects with drugrelated AEs. Adverse events were defined as any untoward medical occurrence and included any clinically relevant findings from physical examination, laboratory tests, ECG, vital-sign measurement and the exacerbation of any preexisting condition. Adverse events were classified according to the following criteria: mild, awareness of signs or symptoms that were easily tolerated; moderate, sufficient discomfort to interfere with usual activities; and severe, incapacitating or causing an inability to work or perform usual activities. Further criteria of interest were: neurological examination; safety laboratory tests (clinical chemistry, haematology and urinalysis); 12-lead ECGs; visual tests (Ishihara test, Jaeger Eye Chart and Amsler grid); ophthalmological examination, including fundus photography, (part 1 only); vital signs [blood pressure, pulse rate and respiratory rate (BI 425809 75-mg BID group only)]; oxygen saturation (BI 425809 75-mg BID group only); suicidality monitoring; and assessment of visual analogue scales (VASs) for possible central nervous system effects (subjective feelings) using the scales developed by Bond and Lader [14] and Bowdle et al. [15].

\subsubsection{Pharmacokinetic Analysis}

The primary pharmacokinetic endpoints (assessed in part 2) were $\mathrm{AUC}_{0-t z}$ and $C_{\text {max }}$ of the analyte. Secondary endpoints assessed in part 1 included AUC of the analyte from 0 to $24 \mathrm{~h}\left(\mathrm{AUC}_{0-24}\right)$ and $C_{\max }$ after the first dose; and AUC and $C_{\max }$ at steady state over a uniform dosing interval $\tau$ 
(AUC $\tau$,ss and $C_{\text {max,ss }}$ ) following the last dose. Additional pharmacokinetic parameters assessed during this study are described within the ESM. The pharmacokinetic software used in this study was Phoenix WinNonlin Version 6.3 (Certara L.P. [Pharsight], St. Louis, MO; for the noncompartmental analysis) using the linear-up log-down trapezoidal method. Statistical analysis software, SAS ${ }^{\circledR}$ Version 9.4 (SAS Institute, Cary, NC, USA), was used to produce graphs and tables.

\subsubsection{Bioanalytical Assay BI 425809 plasma and urine} concentrations were determined by a validated liquid chromatography tandem mass spectrometry assay. The analyses were performed at the Department of Pharmacokinetics and Drug Metabolism, BI Pharmaceuticals Inc., Ridgefield, CT, USA. Linear calibration standard curves (seven concentrations) with $1 / \times 2$ weighting were used to determine BI 425809 concentrations over 1.00-1000 nm. Concentrations below the lower limit of quantification were not replaced by zero at any timepoint (including the lag phase and pre-dose values). For the non-compartmental analysis, concentration values identified as below the lower limit of quantification in the lag phase (period between time zero and the first time point with a concentration above the quantification limit) were set to zero. Back-calculation of calibration standards, tabulation of the standard curve fit parameters and measurement of quality-control samples were used to assess assay performance. The quality-control plasma samples were prepared in K3ethylenediaminetetraacetic acid human plasma, and those for the urine samples were prepared in $0.05 \%$ Tween 20 human urine. Three concentrations were used for both. Quality-control samples were then analysed to assess the accuracy and precision of pharmacokinetic measurements.

\subsection{Statistical Analyses}

Descriptive statistics were calculated for all safety and pharmacokinetic endpoints. Safety was assessed in the treated set, which included all randomised subjects who received one or more doses of the study drug. Pharmacokinetic parameters were assessed in subjects from the treated set who provided one or more evaluable secondary pharmacokinetic endpoint values (pharmacokinetic set). A more detailed description of the statistical analyses can be found in the ESM.

\subsubsection{Part 1: Multiple-Dose Component}

Based on the commonly used dose group size of 12 subjects, it was planned to include 84 subjects in the multipledose component of the study. The size of 12 subjects per dose is often used in multiple rising dose trials of the present type, and is generally considered sufficient for the exploratory evaluation of multiple-dose safety and pharmacokinetics. The pharmacokinetic endpoints $\mathrm{AUC}_{0-24}$, $C_{\text {max }}, \mathrm{AUC}_{\tau, \mathrm{ss}}$ and $C_{\text {max,ss }}$ were assessed for dose proportionality (QD treatment groups only; assessed separately for healthy young and healthy elderly subjects). The linearity index was calculated by building the ratio of $\mathrm{AUC}_{\tau, \mathrm{ss}}$, over $\mathrm{AUC}_{0-\infty}$ and attainment of steady state was assessed based on trough concentrations of BI 425809. Safety endpoints were examined over time and for any differences from baseline values; baseline was defined as the last measurement before the first study-drug administration.

\subsubsection{Part 2: Morning vs. Evening Dosing}

The second part of this study included 12 subjects in order to reach a certain precision in estimating the ratio of gMean with $95 \%$ probability. The effect of evening dosing was determined based on $\mathrm{AUC}_{0-t z}$ and $C_{\max }$, and the related CI. Safety endpoints were examined over time and for any differences from baseline values. Baseline value was considered to be the last measurement before study-drug administration in the morning and the last measurement before study-drug administration in the evening.

\section{Results}

\subsection{Study Population and Disposition}

Ninety-six subjects entered into the study with 93 completing the planned observation time (Fig. 2). Demographic characteristics were similar across all treatment groups (Table 2 of the ESM). Briefly, part 1 of this study included 57 male and 27 female subjects (young and elderly); all except two were Caucasian. Part 2 of the study consisted of ten male and two female healthy young subjects (all Caucasian). Mean (standard deviation) age was 40.5 (8.3) years for young subjects and 71.0 (4.1) years for elderly subjects. During part 1, two subjects discontinued, one young subject after the sixth dose of placebo and one elderly subject after the first dose of BI $42580950 \mathrm{mg}$. During part 2, all except one subject (a healthy young subject discontinued following administration of the single morning dose) completed the planned observation time per protocol.

\subsection{Safety and Tolerability}

A summary of all AEs by system organ class and preferred term ( $\geq 5 \%$ incidence in either part 1 or part 2 ) is provided in Table 1. 


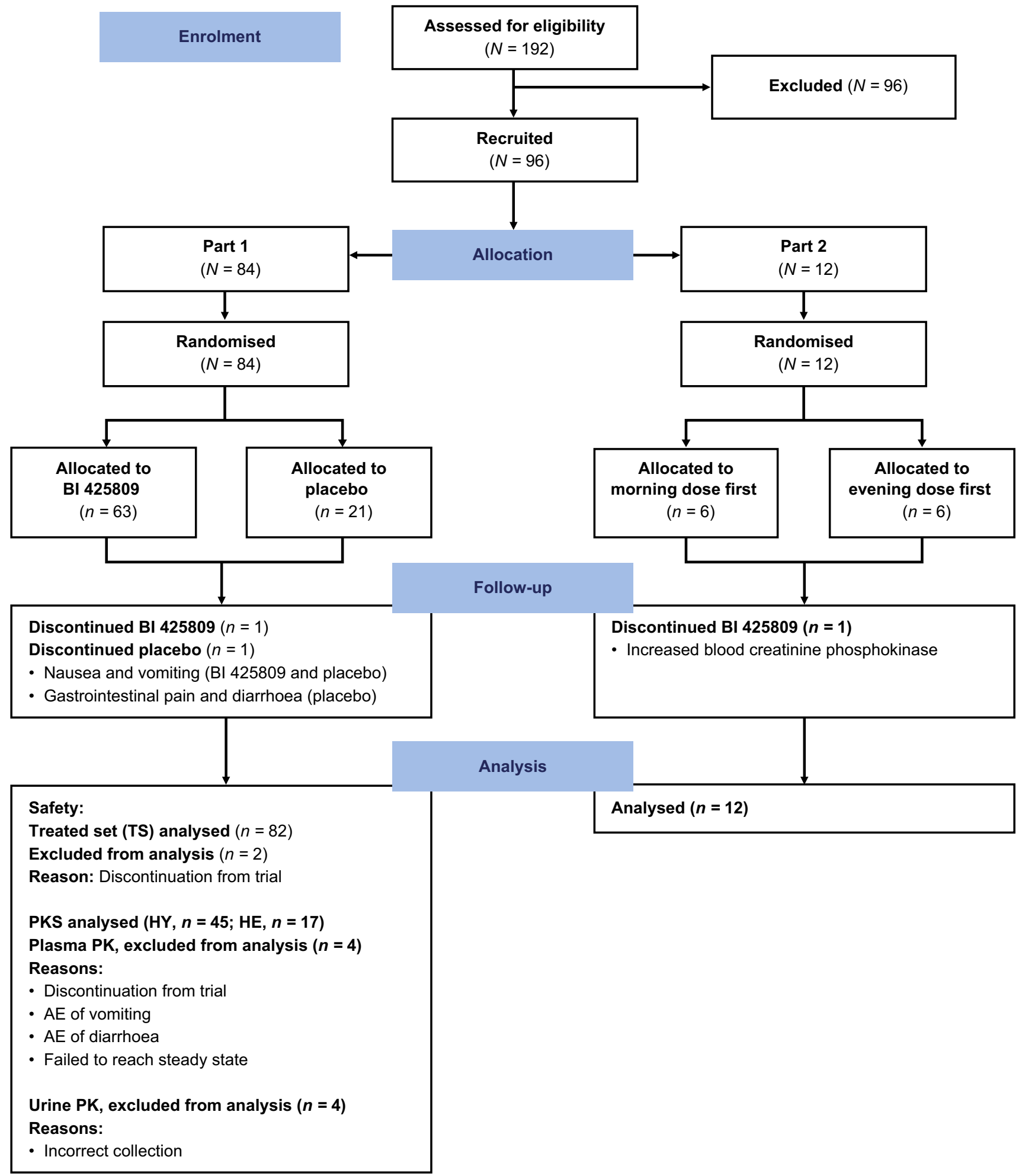

Fig. 2 CONSORT diagram. $A E$ adverse event, $H E$ healthy elderly, $H Y$ healthy young, $P K$ pharmacokinetics, $P K S$ all subjects from the Treated set who received BI 425809, participated in part 1 , and provided one or more PK endpoints judged as evaluable, Treated set all subjects who received at least one dose of study drug/placebo 


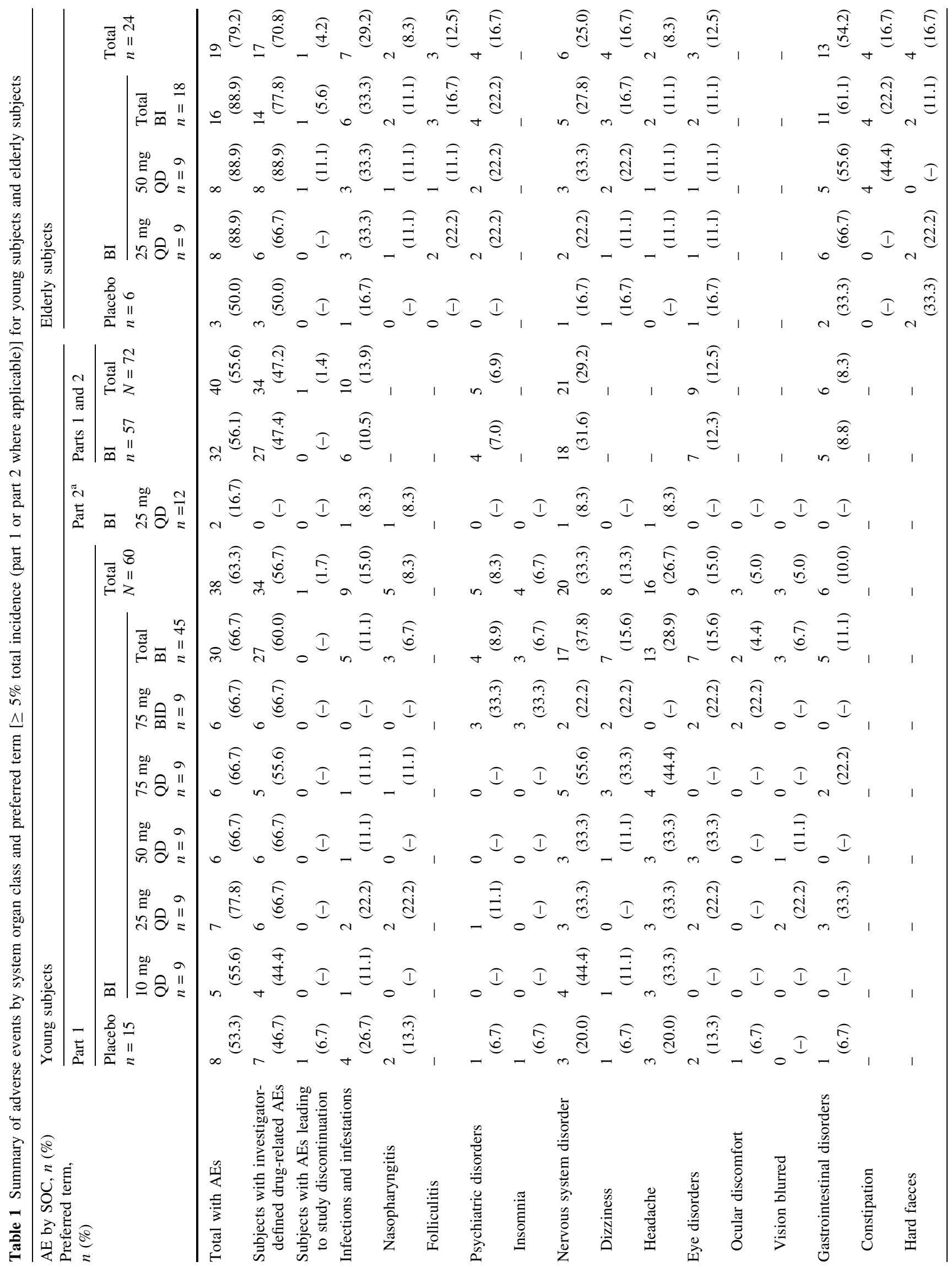




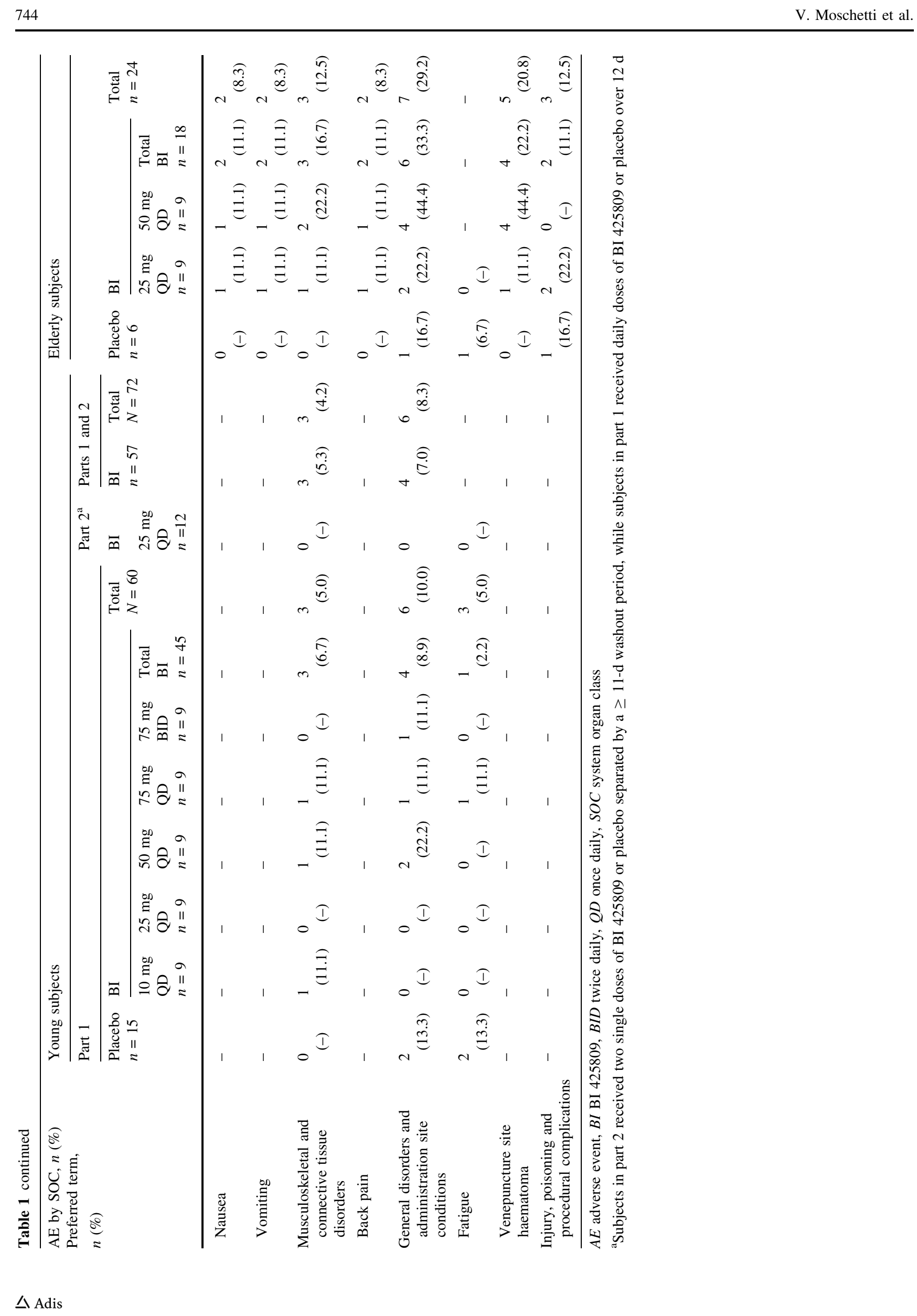




\subsubsection{Part 1: Multiple-Dose Component}

In total, $63.3 \%$ of young subjects experienced one or more AEs: $66.7 \%$ in the active-treatment arm and $53.3 \%$ in the placebo arm. Drug-related AEs were recorded for $60.0 \%$ of subjects receiving BI 425809 and $46.7 \%$ receiving placebo. A greater number of elderly subjects $(79.2 \%)$ than young subjects reported one or more AEs during the study; the incidence was higher for subjects receiving the active drug compared with those receiving placebo (88.9 vs. $50.0 \%$, respectively). As with young subjects, drug-related AEs were reported to a greater extent in elderly subjects who received BI $425809(77.8 \%)$ than those who received placebo $(50.0 \%)$. The overall frequency of subjects with one or more drug-related $\mathrm{AE}$ was similar across all treatment groups, indicating that AEs were not dose dependent (Table 1).

All treatment-emergent AEs were of mild or moderate intensity and no serious AEs were reported. Within parts 1 and 2, the most commonly reported AEs for young subjects in both the active and placebo groups were nervous system disorders, and infections and infestations (system organ class), and headache, dizziness, nasopharyngitis and insomnia (preferred term) (Table 1). In elderly subjects, the most commonly reported AEs in the active-treatment and placebo groups were gastrointestinal disorders (system organ class), and fatigue, constipation, hard faeces, dizziness and venepuncture-site haematoma (preferred term) (Table 1). There were no relevant differences between the treatment groups in the profile of reported AEs.

Both age groups reported eye disorders; all were classified as mild and subjects recovered within a short period of time. Specific events reported by young subjects included ocular discomfort and increased lacrimation (placebo group), visual blurring and impairment, blepharospasm, ocular discomfort, reduced visual acuity and transient reduced visual acuity (active treatment). Elderly subjects reported ocular discomfort (placebo group), blurred vision, visual impairment and reduced visual acuity (active treatment).

No clinically relevant changes in laboratory parameters, vital signs, 12-lead ECG, neurological examinations, ophthalmological examinations, or oxygen saturation monitoring were observed. No suicidal behaviours were noted. Neither VAS revealed clinically relevant dose-related changes in mood state or perception.

\subsubsection{Part 2: Morning vs. Evening Dosing}

Adverse events were reported by two subjects [nasopharyngitis ( $n=1$; duration, days $2-7)$ and headache ( $n=1$; duration, days 1-2)] following a single morning dose of BI $42580925 \mathrm{mg}$. In each case, the AEs were mild or moderate in intensity and considered not to be drug related. No serious AEs were reported. No clinically relevant changes in laboratory parameters, vital signs, 12-lead ECG, neurological examinations, ophthalmological examinations, suicidality monitoring, oxygen saturation monitoring or either VAS were noted.

\subsection{Pharmacokinetic Results}

A full list of secondary pharmacokinetic parameters (part 1) is detailed in Table 3 of the ESM.

\subsubsection{Part 1: Multiple-Dose Component}

BI 425809 was absorbed with median time to maximum plasma concentration values of $3.0-4.5 \mathrm{~h}$ after single as well as multiple doses (Table 2); plasma concentrations subsequently decreased in a biphasic manner. Steady-state conditions were reached between day 6 and day 10 for all treatment groups (i.e. 2-6 days after the start of multiple dosing). Plasma concentration-time profiles were similarly shaped across all dose groups (Fig. 3a) and both age groups (Fig. 3b). Systemic exposure $\left(C_{\max }, C_{\max , s s}, \mathrm{AUC}_{0-24}\right.$ and $\mathrm{AUC}_{\tau, \mathrm{ss}}$ ) demonstrated a trend towards a less-than-doseproportional increase following both single and multiple doses at $50 \mathrm{mg}$ and above (QD) (Table 2). The BI 425809 gMean terminal half-life after the first dose and the terminal half-life at steady state were similar across treatment groups (QD and BID, 34.2-59.3 h), and the fraction eliminated from urine ranged from 1.77 to $2.71 \%$ (after single-dose administration) and from 3.90 to $7.10 \%$ (after multiple dosing). The linearity index, assessed separately for each dose group, was generally close to 1 for BI 425809 QD groups but clearly decreased (0.553) in the 75-mg BID group (Table 3 of the ESM).

Subjects receiving BI $42580925 \mathrm{mg}$ following a standard breakfast (light fed) demonstrated a 23\% increase in steady-state $C_{\max }$ compared with fasted conditions [707 vs. $574 \mathrm{nmol} / \mathrm{L}$; gMean ratio (light fed:fasted), 123.2\%] along with a $14 \%$ increase in steady-state AUC [12,200 vs. $10,700 \mathrm{nmol} \cdot \mathrm{h} / \mathrm{L}$; gMean ratio (light fed:fasted), 114.0\%] and a delayed time to maximum plasma concentration. As expected, subjects showed similar steady-state exposure under light-fasted $\quad\left(\mathrm{C}_{\max }, \quad 662 \mathrm{nmol} / \mathrm{L} ; \quad\right.$ AUC $11,600 \mathrm{nmol} \cdot \mathrm{h} / \mathrm{L})$ and fasted conditions $\left(\mathrm{C}_{\max }, 611 \mathrm{nmol} /\right.$ L; AUC, 11,100 nmol.h/L; gMean ratio [light fasted:fasted] $\mathrm{C}_{\max }, 108.3 \%$; AUC, 104.5\%) [Fig. 1 and Table 4 of the ESM]. Additionally, only slight differences in BI 425809 exposure were identified between light-fed and light-fasted conditions at steady state [gMean ratio (light fed:light fasted) $C_{\max }, 106.8 \%$; AUC, $105.2 \%$ ]. At higher doses $(75 \mathrm{mg})$, single administration of BI 425809 with food (subjects in the BI 425809 BID group) at day 1 led to 
Table 2 Pharmacokinetic parameters after single and multiple doses of BI 425809 during part $1^{\mathrm{a}}$

\begin{tabular}{|c|c|c|c|c|c|c|c|}
\hline \multirow{3}{*}{$\begin{array}{l}\text { Pharmacokinetic } \\
\text { parameter }\end{array}$} & \multicolumn{7}{|l|}{ BI 425809} \\
\hline & \multicolumn{5}{|c|}{ Young subjects } & \multicolumn{2}{|l|}{ Elderly subjects } \\
\hline & $\begin{array}{l}10 \mathrm{mg} \text { QD } \\
n=9\end{array}$ & $\begin{array}{l}25 \mathrm{mg} \text { QD } \\
n=9\end{array}$ & $\begin{array}{l}50 \mathrm{mg} \text { QD } \\
n=9\end{array}$ & $\begin{array}{l}75 \mathrm{mg} \text { QD } \\
n=9\end{array}$ & $\begin{array}{l}75 \mathrm{mg} \mathrm{BID} \\
n=9\end{array}$ & $\begin{array}{l}25 \mathrm{mg} \text { QD } \\
n=8\end{array}$ & $\begin{array}{l}50 \mathrm{mg} \text { QD } \\
n=8\end{array}$ \\
\hline \multicolumn{8}{|c|}{ Single-dose administration } \\
\hline $\mathrm{AUC}_{0-24}, \mathrm{nmol} \cdot \mathrm{h} / \mathrm{L}$ & $1410(20.1)$ & $3720(18.2)$ & $6020(24.4)$ & $7170(17.9)$ & $12,000(13.7)$ & $3460(18.5)$ & $5620(17.9)$ \\
\hline $\begin{array}{l}\mathrm{AUC}_{0-24, \mathrm{norm}} \\
\mathrm{nmol} \cdot \mathrm{h} / \mathrm{L} / \mathrm{mg}\end{array}$ & $141(20.1)$ & $149(18.2)$ & $120(24.4)$ & $95.6(17.9)$ & $160(13.7)$ & $139(18.5)$ & $112(17.9)$ \\
\hline$C_{\max }, \mathrm{nmol} / \mathrm{L}$ & $109(19.0)$ & 278 (19.7) & 397 (28.7) & $451(17.9)$ & $884(8.72)$ & $235(18.7)$ & $371(25.9)$ \\
\hline$C_{\text {max,norm }}, \mathrm{nmol} / \mathrm{L} / \mathrm{mg}$ & $10.9(19.0)$ & $11.1(19.7)$ & $7.95(28.7)$ & $6.01(17.9)$ & $11.8(8.72)$ & $9.4(18.7)$ & $7.43(25.9)$ \\
\hline$t_{\max }^{\mathrm{c}}, \mathrm{h}$ & $4(3.5,5)$ & $4.5(2,5)$ & $4.5(3.5,12)$ & $4(2,5)$ & $4.02(3,5)$ & $3.5(2,5)$ & $4.5(2,8)$ \\
\hline \multicolumn{8}{|c|}{ Multiple-dose administration } \\
\hline $\mathrm{AUC}_{\tau, \mathrm{ss}}^{\mathrm{b}}, \mathrm{nmol} \cdot \mathrm{h} / \mathrm{L}$ & $3870(22.2)$ & $10,600(31.7)$ & $14,200(29.4)$ & $16,500(29.6)$ & $18,800(20.2)$ & $11,000(11.6)$ & $13,900(30.8)$ \\
\hline $\begin{array}{l}\mathrm{AUC}_{\tau, \mathrm{ss}, \mathrm{norm}}{ }^{\mathrm{b}} \\
\mathrm{nmol} \cdot \mathrm{h} / \mathrm{L} / \mathrm{mg}\end{array}$ & $387(22.2)$ & $425(31.7)$ & $285(29.4)$ & $220(29.6)$ & $251(20.2)$ & 439 (11.6) & $278(30.8)$ \\
\hline$C_{\max , \mathrm{ss}}^{\mathrm{b}}, \mathrm{nmol} / \mathrm{L}$ & $221(19.5)$ & $582(27.6)$ & $800(26.3)$ & $1020(23.1)$ & $1930(17.2)$ & $618(10.9)$ & $802(25.0)$ \\
\hline $\begin{array}{l}C_{\max , \mathrm{ss}, \mathrm{norm}} \mathrm{b}, \mathrm{nmol} / \mathrm{L} / \\
\mathrm{mg}\end{array}$ & $22.1(19.5)$ & $23.3(27.6)$ & $16.0(26.3)$ & $13.6(23.1)$ & $25.8(17.2)$ & $24.7(10.9)$ & $16.0(25.0)$ \\
\hline$t_{\max , \mathrm{ss}}^{\mathrm{b}, \mathrm{c}}, \mathrm{h}$ & $4.5(2,5)$ & $3.75(2,6)$ & $3.5(2,6)$ & $4.5(2,6)$ & $3.5(2,4.5)$ & $3(2,4.52)$ & $4.5(3.5,6)$ \\
\hline
\end{tabular}

$A U C_{0-24}$ area under the concentration-time curve from 0 to $24 \mathrm{~h}, A U C_{\tau, s s}$ area under the concentration-time curve at steady state over a uniform dosing interval, $B I D$ twice daily, $C_{\max }$ maximum plasma concentration, $C_{\max , s s}$, maximum plasma concentration at steady state over a uniform dosing interval, $C V$ coefficient of variation, norm dose normalised, $Q D$ once daily, $t_{\max }$, time to $C_{\max }, t_{\max , s s}$ time from last dosing to $C_{\max }$ at steady state over a uniform dosing interval

${ }^{\mathrm{a}} \mathrm{Geometric}$ mean $(\% \mathrm{CV})$, unless otherwise stated

${ }^{\mathrm{b}}$ For dose group BI $42580975 \mathrm{mg} \mathrm{BID}, \mathrm{AUC}_{\tau, 22}, \mathrm{AUC}_{\tau, 22, \text { norm }}, C_{\max , 22}, C_{\max , 22, \text { norm }}$, and $t_{\max , 22}$ are given whereby 22 relates to $22 \mathrm{~h}$

${ }^{\mathrm{c}}$ For $t_{\max }$ and $t_{\mathrm{max}, \mathrm{ss}}$ the median and range (minimum, maximum) are given

a

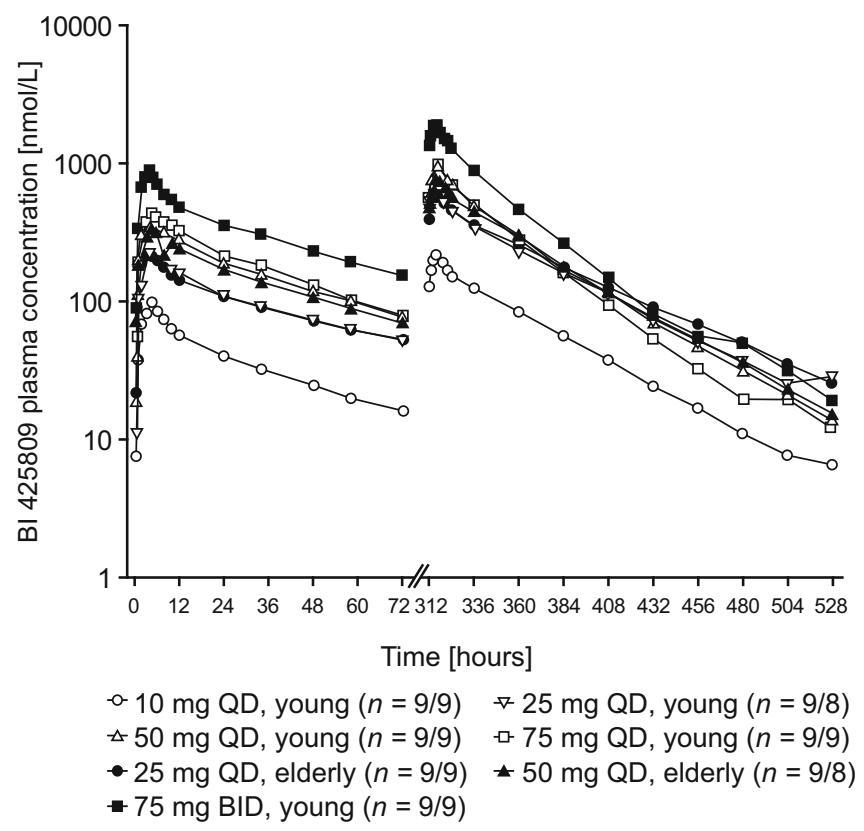

b

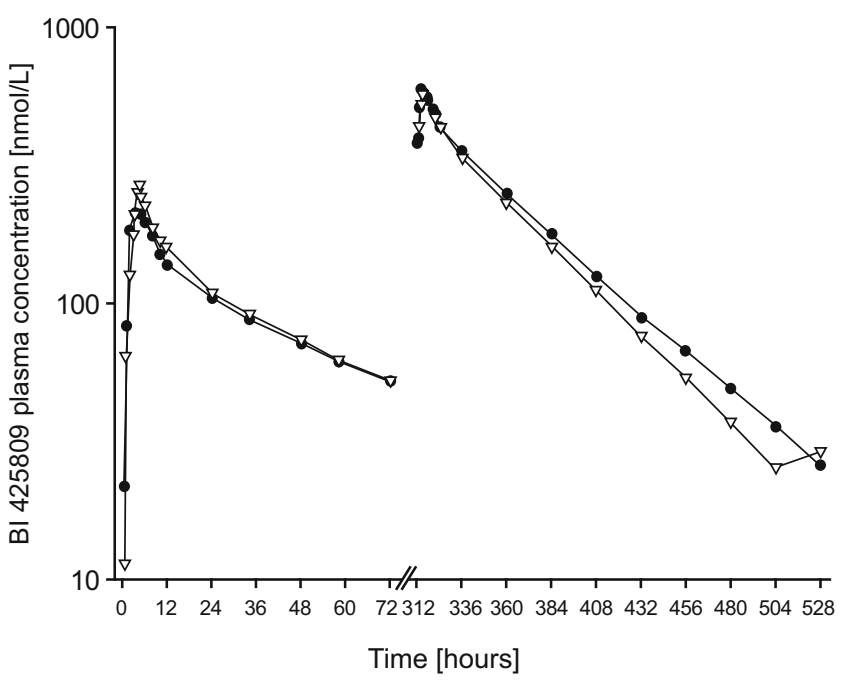

$\rightarrow 25 \mathrm{mg} \mathrm{QD}$, young $(n=9 / 8) \quad \bullet 25 \mathrm{mg} \mathrm{QD}$, elderly $(n=9 / 9)$

Fig. 3 Geometric mean drug plasma concentration-time profiles of BI 425809 after single and multiple oral administration of BI 425809 tablets: a once daily (QD) or twice daily (BID) in young and elderly subjects and b QD in young vs. elderly subjects (semi-log scale) 
increased absorption of BI 425809 compared with fasted conditions (subjects in the BI 425809 QD group). Indeed, $C_{\max }$ and $\mathrm{AUC}_{0-24}$ values approximately doubled [884 nmol/L and 12,000 nmol.h/L (fed); $451 \mathrm{nmol} / \mathrm{L}$ and $7170 \mathrm{nmol} \cdot \mathrm{h} / \mathrm{L}$ (fasted), respectively; Table 2].

\subsubsection{Part 2: Morning vs. Evening Dosing}

Overall, gMean pharmacokinetic parameters suggested a trend towards slightly increased BI 425809 exposure following the evening administration, as suggested by a $15 \%$ higher gMean $C_{\max }(90 \%$ CI 98.1-136) and $20 \%$ higher $\mathrm{AUC}_{0-t z}$ (90\% CI 107-135) compared with morning administration (Table 3). However, when comparing individual $\mathrm{AUC}_{0-t z}$ and $C_{\max }$ values, there appeared to be no systematic trend, with a somewhat higher variability for the morning dosing (Fig. 4).

\section{Discussion}

This study demonstrated that BI 425809 administered orally as multiple rising doses of $10,25,50$ and $75 \mathrm{mg} \mathrm{QD}$, or $75 \mathrm{mg}$ BID in healthy young subjects, or as multiple doses of 25 or $50 \mathrm{mg}$ QD in elderly subjects, over 11 days, was generally well tolerated. All treatment-related AEs were considered of mild or moderate intensity and were in general alignment with recent reports on GlyT1 inhibitors [16-18].

Selective GlyT1 inhibition results in increased synaptic glycine levels, which may be favourable for the treatment of cognitive impairment associated with schizophrenia and $\mathrm{AD}$ [5-7]. Increases in glycine levels, however, have been associated with visual disturbances and electroretinogram alterations [19, 20]. Inhibition of the GlyT1 transporter in the rat retina leads to electroretinogram alterations, which may explain the visual disturbances reported in clinical trials with GlyT1 inhibitors [20]. As such, the visual effects of active BI 425809 administration were extensively monitored throughout this study. Additionally, ophthalmological examination, including fundus photography, was performed. Visual AEs were reported by nine subjects across the treatment groups; all were mild, quickly reversible and did not appear to be dose dependent. Visual analogue scales to monitor possible central nervous system effects (subjective feelings) by evaluating external and internal perception, alertness, mood and calmness were employed during the multiple-dose component of this study and did not reveal any clinically relevant differences between the treatment groups. Overall, the frequency of subjects with one or more drug-related $\mathrm{AE}$ was similar across all treatment groups indicating a lack of dose dependency. Contrary to this, however, administration of BI 425809 as a solution during the previous single rising dose study demonstrated that higher doses were associated with an increased incidence of AEs, the most common being central nervous system and visual effects. This difference may be explained by a greater speed of absorption following administration of BI 425809 as a solution, compared with the tablet formulation [10].

There were no clinically relevant differences in the pharmacokinetics, safety and tolerability between healthy young and healthy elderly subjects during this study. Given that one of the key indications for this therapy will largely consist of elderly patients with $\mathrm{AD}$, the good tolerability of BI 425809 observed within the elderly subjects included here is of particular importance.

Plasma concentration-time profiles of BI 425809 were shaped similarly across all dose groups, and dose proportionality was observed in exposure parameters $\left(C_{\max }\right.$, $\mathrm{AUC}_{0-24}, C_{\mathrm{max}, \mathrm{ss}}$ and $\left.\mathrm{AUC}_{\tau, \mathrm{ss}}\right)$ for BI 42580910 and $25 \mathrm{mg}$. At doses of $\geq 50 \mathrm{mg}$, the increase in exposure was clearly less than dose proportional. By contrast, dose-linear

Table 3 Bioavailability of BI 425809 administered as a 25-mg single morning or evening dose (part 2)

\begin{tabular}{|c|c|c|c|c|}
\hline \multirow[t]{2}{*}{$\begin{array}{l}\text { Pharmacokinetic } \\
\text { parameter }\end{array}$} & \multicolumn{4}{|l|}{$\begin{array}{l}\text { BI } 42580925 \mathrm{mg} \text { QD } \\
N=12\end{array}$} \\
\hline & $\begin{array}{l}\text { Adjusted gMean evening } \\
\text { dose }\end{array}$ & $\begin{array}{l}\text { Adjusted gMean morning } \\
\text { dose }\end{array}$ & $\begin{array}{l}\text { Adjusted gMean ratio } \\
\text { evening/morning } \\
(90 \% \text { CI [\%]) }\end{array}$ & $\begin{array}{l}\text { Intraindividual } \mathrm{gCV}, \\
\%\end{array}$ \\
\hline $\mathrm{AUC}_{0-t z}, \mathrm{nmol} \cdot \mathrm{h} / \mathrm{L}$ & 8410 & 7000 & $120(107-135)$ & 14.6 \\
\hline$C_{\max }, \mathrm{nmol} / \mathrm{L}$ & 278 & 241 & $115(98.1-136)$ & 21.2 \\
\hline $\mathrm{AUC}_{0-\infty}, \mathrm{nmol} \cdot \mathrm{h} / \mathrm{L}$ & 10,400 & 8770 & $118(104-134)$ & 16.1 \\
\hline$t_{\max }{ }^{\mathrm{a}}, \mathrm{h}$ & $4.50(2.00,8.00)$ & $4.00(1.00,4.50)$ & - & - \\
\hline
\end{tabular}

$A U C_{0-t z}$ area under the concentration-time curve over the time interval from 0 to the last quantifiable data point, $A U C_{0-\infty}$ area under the concentration-time curve over the time interval from 0 extrapolated to infinity, $C_{\text {max }}$ maximum plasma concentration, $g C V$ geometric coefficient of variation, gMean geometric mean, $t_{\max }$ time to $C_{\max }$

${ }^{\mathrm{a}}$ For $\mathrm{t}_{\mathrm{max}}$, the median and range (minimum, maximum) are given 


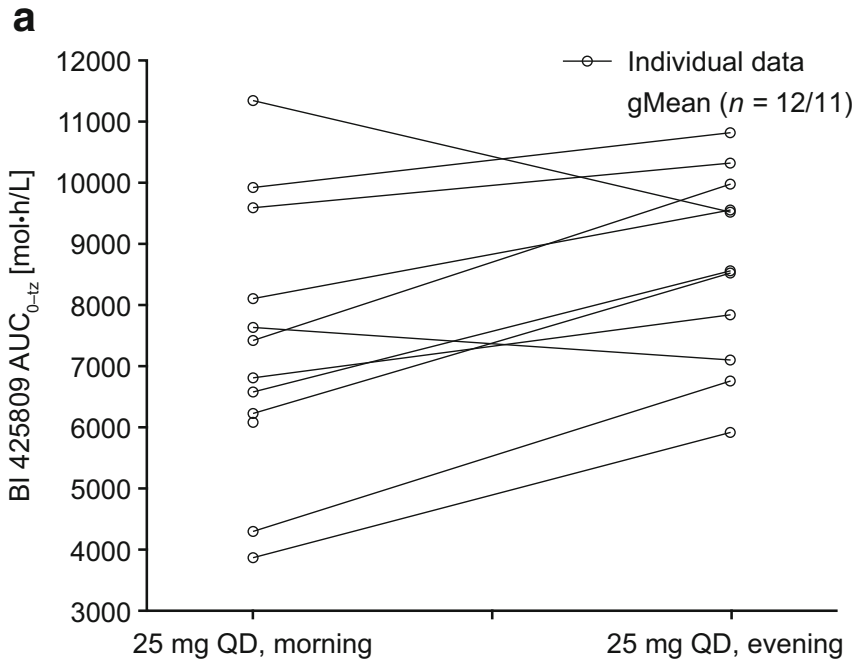

Fig. 4 Intra-individual comparison of a area under the concentration-time curve over the time interval from 0 to the last quantifiable data point $\left(\mathrm{AUC}_{0-t z}\right)$ and $\mathbf{b}$ maximum plasma concentration $\left(C_{\max }\right)$

pharmacokinetics following single administration of BI 425809 as an oral solution from 0.5 to $150 \mathrm{mg}$ has been demonstrated [10], suggesting that the less-than-dose-proportional increase in plasma exposure in the current study can be attributed to the tablet formulation, likely owing to impaired dissolution of the drug from its tablet form rather than effects on absorption or disposition of the drug. Indeed, BI 425809 demonstrates low intrinsic solubility.

Given that only a slight increase in plasma exposure was seen following administration of BI $42580975 \mathrm{mg}$ compared with $50 \mathrm{mg}$ (tablet formulation), it was anticipated that higher QD doses would not achieve further significant increases in plasma exposure. Consequently, a 75-mg BID dosing regimen under fed conditions was introduced to maximise plasma exposure, and thereby the exposure multiplies, in comparison with the maximum anticipated therapeutic dose of $25 \mathrm{mg}$ QD. This approach successfully counteracted the impaired bioavailability of BI 425809 , increasing plasma exposure by $\sim 70 \%$, based on $\mathrm{AUC}_{0-24}$ and $C_{\text {max }}$ after the first dose, compared with the 75-mg QD regimen, an effect that may be attributed to administration of the tablets with food. Nevertheless, such results must be treated with caution given that the comparison was between subject groups rather than intra-individually.

Interestingly, $\mathrm{AUC}_{\tau, \mathrm{ss}}$ increased by just $14 \%$, while $C_{\text {max ss }}$ increased by $90 \%$ following administration of BI $42580975 \mathrm{mg}$ BID vs. $75 \mathrm{mg}$ QD, indicating that after multiple dosing an additional mechanism is impacting plasma exposure. This is further illustrated by a linearity index below 1 for the 75-mg BID dose group, compared with a value close to 1 for the QD treatment groups. Autoinduction of BI 425809 metabolism may be the underlying mechanism behind such observations, as in- b

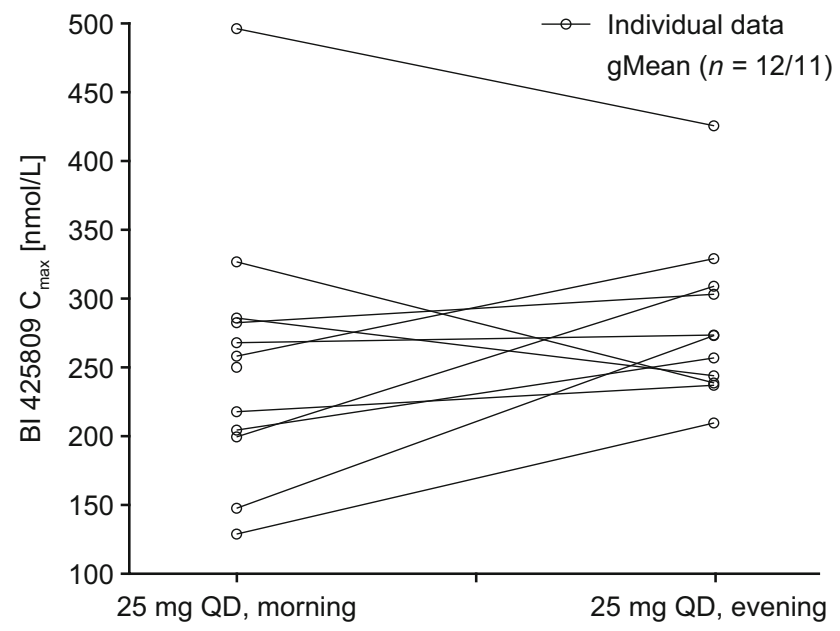

values of BI 425809 after single oral administration of $25 \mathrm{mg}$ in the morning or in the evening in healthy young subjects. gMean geometric mean, $Q D$ once daily

vitro and clinical studies have indicated that BI 425809 is primarily metabolised by CYP3A4, and has the potential to induce CYP3A4 activity [11, 21]. Given that this phenomenon was only apparent for the 75-mg BID dose group, it can be concluded that autoinduction of CYP3A4 at dosing regimens leading to lower exposure is of minor importance.

In summary, these data suggest that decreased bioavailability observed at higher tablet dosing regimens is caused by two factors; low intrinsic solubility and autoinduction. Neither, however, appear to be relevant to the anticipated therapeutic dose range.

An additional factor found to impact upon BI 425809 plasma exposure level is food. A recent investigation demonstrated a $42 \%$ increase in $C_{\max }$, and a $26 \%$ increase in AUC, following a single dose of BI $42580925 \mathrm{mg}$ administered as a tablet with a high-fat/high-calorie meal vs. under fasted conditions [10]. This is in line with the low intrinsic solubility of BI 425809 as it has recently been demonstrated that increased bile secretion, known to accompany high-fat meal consumption, plays an important role in drug accessibility, possibly owing to improved solubility [22]. In the present study, additional exploratory data were generated to characterise the administration of BI 425809 with food.

Administration of BI $42580925 \mathrm{mg}$ in young subjects, in the presence of a standardised light breakfast, led to only a 14 and $23 \%$ increase in steady-state AUC and $C_{\max }$, respectively, compared with fasted conditions. As mentioned above, the food effect seems to be increased ( 70\%) at higher doses, e.g. when comparing single-dose data of the 75-mg dosing regimens. Considering this dose dependency, it may be reasonable to speculate that at doses 
below $25 \mathrm{mg}$ such a food effect may be reduced or even completely eradicated.

It must be noted that the food-effect investigations employed during this study are clearly exploratory in nature; however, they might be closer to the conditions apparent in clinical practice. Because evaluations were partly conducted after multiple dosings with differing food conditions, the preceding doses and conditions might have impacted the food effect readout. Furthermore, the abovementioned factors such as dose dependency of tablet bioavailability and autoinduction at higher doses require a differential consideration of the influence of food on BI 425809 exposure.

Exposure to a BI $42580925-\mathrm{mg}$ tablet was approximately $15-20 \%$ higher $\left[15 \%\right.$ higher gMean $C_{\max }(90 \%$ CI 98.1-136) and 20\% higher $\mathrm{AUC}_{0-\mathrm{tz}}(90 \%$ CI 107-135)] when administered in the evening compared with the morning. Considering an exposure multiple of 3.2-fold when comparing $\mathrm{AUC}_{0-24}$ and $C_{\max }$ between the $25-\mathrm{mg}$ dose group and the well tolerated 75-mg BID dose group, a potential exposure increase by $20 \%$ for the evening dosing is considered not clinically relevant at least with regard to safety. It might be speculated that there is a slight circadian rhythm for the absorption, distribution, metabolism and excretion mechanisms of BI 425809, alternatively this may be owing to procedural differences for the evening vs. morning dosing. Overall, based on current data, the exposure increase of $15-20 \%$ (if a true effect) is not considered clinically relevant, suggesting that either morning or evening doses could be considered for future studies.

\section{Conclusions}

In this phase I, multiple rising-dose study, BI 425809 was well tolerated within the tested dose range in healthy young and elderly subjects. Within the predicted therapeutic exposure range of $\mathrm{BI} 425809 \leq 25 \mathrm{mg}$ QD, pharmacokinetic parameters demonstrated no obvious deviation from dose linearity.

Acknowledgements The authors thank Jakub Pekarek and Marion Schmid for their technical support of the pharmacokinetic analyses, and Jasmin Link for her review of the manuscript.

\section{Compliance with Ethical Standards}

Funding The work presented here, including the conduct of the study, data analysis and interpretation, was funded by Boehringer Ingelheim. The sponsor was given the opportunity to review the manuscript for medical and scientific accuracy as well as intellectual property considerations. Editorial support in the form of initial preparation of the outline based on input from all authors, and collation and incorporation of author feedback to develop subsequent drafts, assembling tables and figures, copy editing and referencing was provided by Louisa Pettinger, $\mathrm{PhD}$, and Sam Halliwell, $\mathrm{PhD}$, of Fishawack Communications, and was funded by Boehringer Ingelheim International $\mathrm{GmbH}$.

Conflict of interest The authors met the criteria for authorship as recommended by the International Committee of Medical Journal Editors. All authors (except Armin Schultz, who is an employee of CRS Clinical Research Services, Mannheim) are employees of Boehringer Ingelheim, but received no direct compensation related to the development of this manuscript. Armin Schultz has no disclosures to declare.

Ethics approval All procedures performed in studies involving human participants were in accordance with the ethical standards of the institutional and/or national research committee and with the 1964 Helsinki Declaration and its later amendments or comparable ethical standards.

Consent to participate Informed consent was obtained from all individual participants included in the study.

Open Access This article is distributed under the terms of the Creative Commons Attribution-NonCommercial 4.0 International License (http://creativecommons.org/licenses/by-nc/4.0/), which permits any noncommercial use, distribution, and reproduction in any medium, provided you give appropriate credit to the original author(s) and the source, provide a link to the Creative Commons license, and indicate if changes were made.

\section{References}

1. Evans JD, Bond GR, Meyer PS, et al. Cognitive and clinical predictors of success in vocational rehabilitation in schizophrenia. Schizophr Res. 2004;70(2-3):331-42.

2. Kurtz MM, Wexler BE, Fujimoto M, et al. Symptoms versus neurocognition as predictors of change in life skills in schizophrenia after outpatient rehabilitation. Schizophr Res. 2008;102(1-3):303-11.

3. Velligan DI, Mahurin RK, Diamond PL, et al. The functional significance of symptomatology and cognitive function in schizophrenia. Schizophr Res. 1997;25(1):21-31.

4. Green MF, Kern RS, Braff DL, Mintz J. Neurocognitive deficits and functional outcome in schizophrenia: are we measuring the "right stuff"? Schizophr Bull. 2000;26(1):119-36.

5. Goff DC, Coyle JT. The emerging role of glutamate in the pathophysiology and treatment of schizophrenia. Am J Psychiatry. 2001;158(9):1367-77.

6. Danysz W, Parsons CG. Alzheimer's disease, beta-amyloid, glutamate, NMDA receptors and memantine: searching for the connections. Br J Pharmacol. 2012;167(2):324-52.

7. Hashimoto K. Glycine transport inhibitors for the treatment of schizophrenia. Open Med Chem J. 2010;4:10-9.

8. Cooke SF, Bliss TV. Long-term potentiation and cognitive drug discovery. Curr Opin Investig Drugs. 2005;6(1):25-34.

9. Collingridge GL, Volianskis A, Bannister N, et al. The NMDA receptor as a target for cognitive enhancement. Neuropharmacology. 2013;64:13-26.

10. Moschetti V, Desch M, Goetz S, et al. Safety, tolerability and pharmacokinetics or oral BI 425809, a glycine transporter 1 inhibitor, in healthy male volunteers: a partially randomised, single-blind, placebo-controlled, first-in-human study. Eur J Drug Metab Pharmacokinet. 2018;43(2):239-49. 
11. Desch M, Goettel M, Goetz S, et al. Effects of the potent cytochrome p450 3A4 inhibitor, itraconazole, on the pharmacokinetics of BI 425809, a new glycine transporter 1 (GlyT1) inhibitor. Clin Pharmacol Ther. 2017;101(S1):S52.

12. International Council For Harmonisation Of Technical Requirements For Pharmaceuticals For Human Use (ICH). ICH harmonised guideline. Integrated addendum to ICH E6 (R1): guideline for good clinical practice E6 (R2). Current Step 4 version; 9 Nov 2016.

13. World Medical Association. World Medical Association Declaration of Helsinki: ethical principles for medical research involving human subjects. JAMA. 2013;310(20):2191-4.

14. Bond $\mathrm{A}$, Lader $\mathrm{M}$. The use of analogue scales in rating subjective feelings. Br J Med Psychol. 1974;47(3):211-8.

15. Bowdle TA, Radant AD, Cowley DS, et al. Psychedelic effects of ketamine in healthy volunteers: relationship to steady-state plasma concentrations. Anesthesiology. 1998;88(1):82-8.

16. D'Souza DC, Singh N, Elander J, et al. Glycine transporter inhibitor attenuates the psychotomimetic effects of ketamine in healthy males: preliminary evidence. Neuropsychopharmacology. 2012;37(4):1036-46.

17. Hirayasu Y, Sato S-I, Takahashi H, et al. A double-blind randomized study assessing safety and efficacy following one-year adjunctive treatment with bitopertin, a glycine reuptake inhibitor, in Japanese patients with schizophrenia. BMC Psychiatry. 2016;16(1): 1

18. Umbricht D, Alberati D, Martin-Facklam M, et al. Effect of bitopertin, a glycine reuptake inhibitor, on negative symptoms of schizophrenia: a randomized, double-blind, proof-of-concept study. JAMA Psychiatry. 2014;71(6):637-46.

19. Liem-Moolenaar M, Peeters P, Kamerling IMC, et al. Early stage development of the glycine-1 re-uptake inhibitor SCH 900435: central nervous system effects compared with placebo in healthy men. Br J Clin Pharmacol. 2013;75(6):1455-67.

20. Liu CN, Pettersen B, Seitis G, et al. GlyT1 inhibitor reduces oscillatory potentials of the electroretinogram in rats. Cutan Ocul Toxicol. 2014;33(3):206-11.

21. Desch M, Schmitt H, Hohl K, et al. Pharmacokinetic interaction of BI 425809, a new glycine transporter 1 (GlyT1) inhibitor, with cytochrome p450 (CYP) isoenzymes and p-glycoprotein (P-gp) probe drug. Clin Pharmacol Ther. 2017;101(S1):S52.

22. Lyng E, Havenaar R, Shastri P, et al. Increased bioavailability of celecoxib under fed versus fasted conditions is determined by postprandial bile secretion as demonstrated in a dynamic gastrointestinal model. Drug Dev Ind Pharm. 2016;42(8):1334-9. 